\title{
Lipopolysaccharide binding protein, obesity status and incidence of metabolic syndrome: a prospective study among middle-aged and older Chinese
}

\author{
Xin Liu • Ling Lu • Pang Yao • Yiwei Ma • Feijie Wang • \\ Qianlu Jin • Xingwang Ye • Huaixing Li • Frank B. Hu • \\ Liang Sun $\cdot$ Xu Lin
}

Received: 16 February 2014 / Accepted: 14 May 2014 /Published online: 8 June 2014

(C) Springer-Verlag Berlin Heidelberg 2014

\begin{abstract}
Aims/hypothesis Although microbiota-derived endotoxaemia has previously been shown to induce metabolic disorders, data from population-based longitudinal studies are scarce. This study therefore investigated the associations between lipopolysaccharide binding protein (LBP) levels and 6 year incident metabolic syndrome (MetS), as well as the potentially modifying effects of obesity status in middle-aged and older Chinese men and women.

Methods A total of 2,529 men and women aged 50-70 years from Beijing and Shanghai, China, were followed for 6 years. Those free of MetS at baseline $(1,312)$ were included in the analyses for the risk of developing MetS. Baseline plasma LBP was measured using an ELISA kit.
\end{abstract}

X. Liu and L. Lu contributed equally to this work.

Electronic supplementary material The online version of this article (doi:10.1007/s00125-014-3288-7) contains peer-reviewed but unedited supplementary material, which is available to authorised users.

X. Liu $\cdot$ L. Lu $\cdot$ P. Yao $\cdot$ Y. Ma $\cdot$ F. Wang $\cdot$ Q. Jin $\cdot$ X. Ye $\cdot$ H. Li $\cdot$

L. Sun $(\triangle) \cdot X$. Lin $(\varangle)$

Key Laboratory of Nutrition and Metabolism, Institute for Nutritional Sciences, Shanghai Institutes for Biological Sciences, Chinese Academy of Sciences and University of Chinese Academy of Sciences, 320 Yueyang Road, Shanghai 200031, People's Republic of China

e-mail: sunliang@sibs.ac.cn

e-mail: xlin@sibs.ac.cn

F. B. Hu

Department of Nutrition and Epidemiology, Harvard School of

Public Health, Boston, MA, USA

F. B. $\mathrm{Hu}$

Channing Division of Network Medicine, Department of Medicine, Brigham and Women's Hospital and Harvard Medical School, Boston, MA, USA
Results During the 6 year follow-up, 449 (34.2\%) participants developed MetS. Baseline LBP was significantly associated with BMI, waist circumference, blood lipid profile and C-reactive protein (CRP) both at baseline and during follow-up (all $p<0.05)$. The RR for incident MetS comparing extreme quartiles of LBP was 1.28 (95\% CI 1.04, 1.58), after multivariate adjustment including BMI and CRP. In stratified analysis, LBP was positively associated with incident MetS only in normal-weight participants (RR, comparing extreme tertiles, 1.59 ; 95\% CI 1.18, $2.15 ; p_{\text {trend }}=0.002$ ), but not in their overweight/obese counterparts (RR, comparing extreme tertiles, 0.99; 95\% CI 0.80, 1.22; $p$ trend $=0.880)$. A significant interaction was observed between LBP and obesity status ( $p_{\text {interaction }}=0.013$ ).

Conclusions/interpretation Our study suggested that elevated plasma LBP was associated with an increased risk of developing MetS among middle-aged and older Chinese, especially in normal-weight individuals.

Keywords Lipopolysaccharide binding protein · Metabolic syndrome $\cdot$ Obesity $\cdot$ Prospective cohort study

$\begin{array}{ll}\text { Abbreviations } \\ \text { CD14 } & \text { Cluster of differentiation } 14 \\ \text { CRP } & \text { C-reactive protein } \\ \text { DNL } & \text { De novo lipogenesis } \\ \text { GGT } & \gamma \text {-Glutamyl transpeptidase } \\ \text { LBP } & \text { Lipopolysaccharide binding protein } \\ \text { LPS } & \text { Lipopolysaccharide } \\ \text { MetS } & \text { Metabolic syndrome } \\ \text { PAI-1 } & \text { Plasminogen activator inhibitor 1 } \\ \text { RBP-4 } & \text { Retinol binding protein } 4 \\ \text { TLR4 } & \text { Toll-like receptor } 4 \\ \text { WC } & \text { Waist circumference }\end{array}$




\section{Introduction}

An increasing number of human studies have supported a link between certain gut microbiota signatures and obesity-related disorders, including hyperlipidaemia, insulin resistance and type 2 diabetes [1-3]. However, it is still unclear how microbiota interacts with the human body and, consequently, how it affects metabolic homeostasis. One of the most important crosstalks between gut microbiota and humans is recognised as 'endotoxaemia', characterised by elevated circulating lipopolysaccharide (LPS), an endotoxin produced by Gramnegative bacteria [4]. Intravenous infusion of LPS in an acute human experimental study was found to induce systemic insulin resistance and elevation of inflammatory markers in adipose tissue [5]. However, owing to its short half-life and interference in the blood, it is difficult to measure circulating LPS in large-scale population-based studies [6]. By contrast, lipopolysaccharide binding protein (LBP), which is mainly synthesised in the liver, could specifically bind to and monomerise exogenous LPS, thereafter enabling the endotoxin to be recognised by Toll-like receptor 4 (TLR4) and cluster of differentiation 14 (CD14), which are responsible for consequent innate immunity [7]. Therefore, circulating LBP levels, with a relatively long half-life, have been used as a variable to assess endotoxaemia status and its immune responses $[8,9]$.

In a previous case-control study, we observed that increased plasma LBP was associated with a higher prevalence of metabolic syndrome (MetS) and type 2 diabetes, but these associations were attenuated considerably when controlling for inflammatory markers [9], suggesting that gut microbiota might lead to human metabolic abnormalities by triggering chronic inflammation through LPS invasion [4]. On the other hand, the components of MetS, such as hypertriacylglycerolaemia, hyperglycaemia and hyperinsulinaemia, have been hypothesised to contribute to endotoxaemia through altering jejunal motility and gastrointestinal transit time, conditions that may favour bacterial overgrowth and leak endotoxins into the intestinal mucosa $[10,11]$. Whether endotoxaemia predisposes to the onset of metabolic deterioration in free-living populations remains to be elucidated. Population-based prospective studies regarding endotoxaemia and metabolic diseases are scarce.

It has been found that obese individuals have significantly higher LBP levels compared with their normal-weight counterparts $[9,12]$. Moreover, a recent study from Spain suggested a significant correlation between gene expressions of LBP and gene expressions related to inflammation and insulin resistance in adipose tissue [13]. Hence, adiposity may play a role in the putative endotoxaemia-metabolic axis, although LBP is mainly derived from liver. The current study aimed to investigate: (1) the association between plasma LBP and the 6 year incidence of MetS; and (2) potential modification effects of obesity status on the LBP-MetS association in middle-aged and older Chinese individuals.

\section{Methods}

Study population The study population was from the Nutrition and Health of Aging Population in China, a population-based prospective cohort study of 3,289 adults aged 50-70 years in Beijing and Shanghai, China. A detailed study design has been described elsewhere [14]. Briefly, the study was initiated in 2005 and designed to assess the impacts of environmental and genetic factors, as well as their interactions, on metabolic diseases. In 2011, 2,529 participants were enrolled in a follow-up visit. When analysing the RR of MetS, participants were excluded if they met any of the following criteria: (1) having MetS at baseline; (2) having insufficient data to define incident MetS; (3) having no baseline LBP data; or (4) having presumed acute inflammation (C-reactive protein $[\mathrm{CRP}]>95.2 \mathrm{nmol} / 1[10 \mathrm{mg} / \mathrm{l}])$. For analyses of the incidence of each MetS component, individuals with a given MetS component at baseline were excluded. The numbers of participants in each analysis were therefore: 1,312 for MetS, 1,151 for central obesity, 1,702 for hypertriacylglycerolaemia, 1,345 for hyperglycaemia, 700 for elevated blood pressure and 1,305 for low HDL-cholesterol. The study protocol was approved by the Institutional Review Board of the Institute for Nutritional Sciences, Chinese Academy of Sciences. Written informed consent was obtained from all participants.

Data collection Information on demography, health status and lifestyle factors was collected using a standard questionnaire. Body weight was measured to the nearest $0.1 \mathrm{~kg}$ and height was measured to the nearest $0.1 \mathrm{~cm}$, with all participants dressed in light clothing and without shoes. BMI was calculated as weight in kilograms divided by the square of height in metres. Waist circumference (WC) was measured at the midpoint between the lowest rib and the iliac crest to the nearest $0.1 \mathrm{~cm}$, after inhalation and exhalation. After a minimum rest of $5 \mathrm{~min}$, blood pressure was measured three times in the right arm using an electronic blood pressure monitor (Omron HEM-705CP; Omron Healthcare, Vernon Hills, IL, USA), and the average of the last two measurements was used in the analyses.

Laboratory measurements Blood samples were collected after an overnight fast both at baseline and follow-up visits. Clinical biomarkers including glucose, triacylglycerol, HDLcholesterol, LDL-cholesterol and CRP were measured on an automatic analyser (Hitachi 7080; Hitachi, Tokyo, Japan). $\mathrm{HbA}_{1 \mathrm{c}}$ was measured using an automated immunoassay (Tina-Quant Hemoglobin A1C II; Roche Diagnostics, Indianapolis, IN, USA) and was standardised according to 
the Diabetes Control and Complications Trial/National Glycohemoglobin Standardization Program. Insulin level was determined by radioimmunoassay (for baseline: LINCO Research, St Charles, MO, USA; for the follow-up visit: Merck Millipore, Billerica, MA, USA). The HOMA-IR was calculated according to the updated HOMA model (www.dtu. ox.ac.uk/). Baseline plasma LBP was measured using an ELISA kit (Hycult Biotech, Uden, the Netherlands), according to the manufacturer's instructions. The intra- and inter-assay $\mathrm{CVs}$ were $<10 \%$ and $<15 \%$, respectively. Measurements of baseline plasma levels of retinol binding protein 4 (RBP-4), ferritin, gamma-glutamyl transpeptidase (GGT), resistin, adiponectin, plasminogen activator inhibitor 1 (PAI-1), TNF- $\alpha$ receptor 2 and erythrocyte palmitoleic acid have been previously described [14-16].

Definition of outcomes Overweight was defined as BMI $\geq 24 \mathrm{~kg} / \mathrm{m}^{2}$ and $\mathrm{BMI}<28 \mathrm{~kg} / \mathrm{m}^{2}$, and obesity was defined as BMI $\geq 28 \mathrm{~kg} / \mathrm{m}^{2}$, according to the recommendation of the Cooperative Meta-Analysis Group of the Working Group on Obesity in China [17]. MetS was defined according to the updated National Cholesterol Education Program Panel III criteria for Asian-Americans [18] if participants had at least three of the following components: (1) $\mathrm{WC} \geq 90 \mathrm{~cm}$ for men or $\geq 80 \mathrm{~cm}$ for women (central obesity); (2) triacylglycerol $\geq 1.7 \mathrm{mmol} / \mathrm{l}$ (hypertriacylglycerolaemia); (3) HDL-cholesterol $<1.03 \mathrm{mmol} / \mathrm{l}$ for men or $<1.30 \mathrm{mmol} / 1$ for women; (4) blood pressure $\geq 130 / 85 \mathrm{mmHg}$ or current use of antihypertensive drugs; (5) fasting glucose $\geq 5.6 \mathrm{mmol} / \mathrm{l}$ or previous diagnosis of type 2 diabetes or taking oral glucose-lowering agents or insulin.

Statistical analyses The comparisons of baseline characteristics according to quartiles of LBP were tested by analysis of covariance for continuous variables and logistic regression for categorical variables. The correlation coefficients between LBP level and metabolic traits were calculated by partial correlation analysis on ranks (Spearman correlation) after adjustment for age, sex, region (north, south) and residence (rural, urban). Owing to the high incidence of MetS in the current population, log Poisson regression was used to estimate the RRs and CIs [19]. Potential confounding factors included age, sex, region, residence, current drinking (yes, no), current smoking (yes, no), physical activity (low, moderate or high), educational attainment ( $<6$ years, $7-9$ years or $\geq 10$ years) and family history of chronic diseases (a parent or first-degree sibling having any of the following conditions: coronary heart disease, stroke, hypertension or diabetes). Stratified analyses were performed at different baseline levels of age, sex, BMI and CRP. The $p$ value for interaction was calculated using a likelihood ratio test. All the statistical analyses were conducted using Stata 9.2 (StataCorp LP, College Station, TX, USA). The significance level was set as two-sided $p<0.05$.

\section{Results}

Baseline characteristics Baseline characteristics according to quartiles of LBP and BMI categories are shown in Table 1 and electronic supplementary material (ESM) Table 1, respectively. Participants with higher levels of LBP were more likely to be rural and southern residents and to have lower educational attainment. Moreover, increased plasma LBP was associated with higher levels of BMI, WC, triacylglycerol, triacylglycerol/HDLcholesterol ratio and CRP, as well as lower concentrations of HDL-cholesterol. In general, overweight/obese individuals had higher levels of LBP, CRP, insulin and HOMA-IR, and an adverse lipid profile, compared with their normal-weight counterparts.

Correlations between baseline LBP and metabolic traits at baseline and follow-up Overall, baseline LBP was significantly correlated with BMI, WC, triacylglycerol, HDLcholesterol, triacylglycerol/HDL-cholesterol ratio and $\mathrm{CRP}$ at baseline and during follow-up visits, after adjustment for age, sex, region and residence $(p<0.05)$ (Table 2). Moreover, LBP was also correlated with $\mathrm{HbA}_{1 \mathrm{c}}$ at baseline, and with glucose, total and LDLcholesterol, blood pressure and HOMA-IR at follow-up $(p<0.05)$. When stratified by obesity status, most of the correlations remained significant only in the normalweight participants, but not in the overweight/obese participants, whose LBP was only correlated with CRP at baseline and follow-up, and with total and LDLcholesterol at baseline only. Furthermore, baseline LBP levels were also significantly correlated with ferritin, resistin, PAI-1 and erythrocyte palmitoleic acid at baseline, but not with RBP-4, GGT, adiponectin or TNF- $\alpha$ receptor 2, after adjustment for age, sex, region and residence (ESM Table 2). The correlations were only significant for PAI-1 and palmitoleic acid $(p<0.05)$ when further controlling for CRP.

Associations between plasma LBP and incident MetS and its components At the end of the 6 year follow-up, a total of 449 (34.2\%) participants developed MetS. Higher baseline LBP levels were significantly associated with the increasing number of MetS components at follow-up $(p<0.001 ;$ Fig. 1$)$. After adjustment for age, sex, region, residence, drinking, smoking, education, physical activity and family history of chronic diseases at baseline (Table 3, Model 1), the RR of MetS was 1.52 (95\% CI $1.23,1.89)$, comparing extreme quartiles of LBP $(p$ trend $<0.001)$. The association was attenuated, but remained significant after further adjustment for BMI (RR $1.30 ; 95 \%$ CI 1.06, 1.59; $p$ trend $=0.023$ ) (Model 2). Additional controlling for baseline CRP levels had no substantial influence on the association (Model 3). When 
Table 1 Baseline characteristics of participants according to quartiles of LBP

\begin{tabular}{|c|c|c|c|c|c|}
\hline \multirow[t]{2}{*}{ Variable } & \multicolumn{4}{|l|}{ Quartile of LBP } & \multirow[t]{2}{*}{$p$ value } \\
\hline & $\mathrm{Q} 1(n=328)$ & $\mathrm{Q} 2(n=328)$ & $\mathrm{Q} 3(n=328)$ & $\mathrm{Q} 4(n=328)$ & \\
\hline $\mathrm{LBP}, \mu \mathrm{g} / \mathrm{ml}$ & $6.62 \pm 1.35$ & $9.51 \pm 0.65$ & $11.59 \pm 0.64$ & $16.18 \pm 3.65$ & $<0.001$ \\
\hline Age, years & $57.2 \pm 5.8$ & $58.0 \pm 5.7$ & $58.4 \pm 6.0$ & $58.0 \pm 6.2$ & 0.052 \\
\hline Male, $n(\%)$ & $160(48.7)$ & $141(43.0)$ & $158(48.2)$ & $165(50.3)$ & 0.429 \\
\hline Urban residents, $n(\%)$ & $162(49.4)$ & $115(35.1)$ & $106(32.3)$ & $136(41.5)$ & 0.028 \\
\hline Northern residents, $n(\%)$ & $182(55.5)$ & $123(37.5)$ & $116(35.4)$ & $140(42.7)$ & $<0.001$ \\
\hline Current smoking, $n(\%)$ & $103(31.4)$ & $92(28.1)$ & $111(33.8)$ & $98(29.9)$ & 0.915 \\
\hline Current drinking, $n(\%)$ & $113(34.5)$ & $90(27.4)$ & $96(29.3)$ & $93(28.4)$ & 0.146 \\
\hline Educational level & & & & & 0.011 \\
\hline $0-6$ years, $n(\%)$ & $121(36.9)$ & $167(50.9)$ & $185(56.4)$ & $151(46.0)$ & \\
\hline 7-9 years, $n(\%)$ & $134(40.9)$ & $92(28.1)$ & $92(28.1)$ & $114(34.8)$ & \\
\hline$\geq 10$ years, $n(\%)$ & $73(22.3)$ & $69(21.0)$ & $51(15.6)$ & $63(19.2)$ & \\
\hline Physical activity level & & & & & 0.918 \\
\hline Low, $n(\%)$ & $24(7.3)$ & $22(6.7)$ & $25(7.6)$ & $16(4.9)$ & \\
\hline Moderate, $n(\%)$ & $123(37.5)$ & $104(31.7)$ & $112(34.2)$ & $129(39.3)$ & \\
\hline High, $n(\%)$ & $181(55.2)$ & $202(61.6)$ & $191(58.2)$ & $183(55.8)$ & \\
\hline Family history of chronic diseases ${ }^{\mathrm{a}}, n(\%)$ & $174(53.1)$ & $173(52.7)$ & $149(45.4)$ & $174(53.0)$ & 0.553 \\
\hline BMI, $\mathrm{kg} / \mathrm{m}^{2}$ & $22.6 \pm 2.8$ & $22.5 \pm 2.8$ & $23.0 \pm 3.0$ & $23.3 \pm 2.9$ & $<0.001$ \\
\hline $\mathrm{WC}, \mathrm{cm}$ & $77.9 \pm 8.7$ & $77.4 \pm 7.9$ & $78.9 \pm 9.7$ & $80.5 \pm 9.1$ & $<0.001$ \\
\hline Systolic blood pressure, $\mathrm{mmHg}$ & $132.2 \pm 22.0$ & $132.8 \pm 19.9$ & $132.6 \pm 20.4$ & $134.6 \pm 21.2$ & 0.175 \\
\hline Diastolic blood pressure, $\mathrm{mmHg}$ & $77.4 \pm 10.7$ & $77.1 \pm 9.9$ & $77.0 \pm 10.5$ & $77.8 \pm 10.2$ & 0.619 \\
\hline Glucose, $\mathrm{mmol} / 1$ & $5.30(4.97,5.67)$ & $5.26(4.92,5.55)$ & $5.22(4.82,5.47)$ & $5.26(4.94,5.56)$ & 0.137 \\
\hline $\mathrm{HbA}_{1 \mathrm{c}}, \%$ & $5.59(5.37,5.85)$ & $5.60(5.39,5.90)$ & $5.65(5.39,5.95)$ & $5.73(5.43,6.05)$ & 0.231 \\
\hline $\mathrm{HbA}_{1 \mathrm{c}}, \mathrm{mmol} / \mathrm{mol}$ & $37.6(35.2,40.4)$ & $37.7(35.4,40.9)$ & $38.2(35.4,41.5)$ & $39.1(35.8,42.6)$ & 0.231 \\
\hline Triacylglycerol, mmol/1 & $0.84(0.63,1.11)$ & $0.83(0.59,1.10)$ & $0.85(0.62,1.56)$ & $0.93(0.69,1.26)$ & 0.006 \\
\hline Total cholesterol, mmol/1 & $4.61 \pm 0.92$ & $4.50 \pm 0.94$ & $4.52 \pm 0.86$ & $4.58 \pm 0.87$ & 0.730 \\
\hline HDL-cholesterol, mmol/1 & $1.46 \pm 0.36$ & $1.40 \pm 0.33$ & $1.40 \pm 0.30$ & $1.36 \pm 0.32$ & $<0.001$ \\
\hline LDL-cholesterol, mmol/1 & $3.15 \pm 0.92$ & $3.05 \pm 0.93$ & $3.08 \pm 0.88$ & $3.15 \pm 0.86$ & 0.858 \\
\hline Triacylglycerol/HDL-cholesterol ratio & $0.57(0.40,0.88)$ & $0.59(0.42,0.86)$ & $0.62(0.34,0.88)$ & $0.70(0.46,1.03)$ & $<0.001$ \\
\hline $\mathrm{CRP}, \mathrm{nmol} / \mathrm{l}$ & $3.05(1.14,5.33)$ & $3.52(1.14,6.43)$ & $4.48(2.57,9.43)$ & $8.43(4.38,19.24)$ & $<0.001$ \\
\hline Insulin, $\mathrm{pmol} / 1$ & $80.0(57.9,104.1)$ & $84.3(60.9,113.5)$ & $82.0(60.9,110.4)^{\mathrm{b}}$ & $82.2(62.8,109.4)^{\mathrm{b}}$ & 0.955 \\
\hline HOMA-IR & $1.52(1.10,1.96)^{\mathrm{b}}$ & $1.60(1.15,2.17)$ & $1.54(1.16,2.08)^{\mathrm{b}}$ & $1.56(1.18,2.04)^{\mathrm{b}}$ & 0.398 \\
\hline
\end{tabular}

Values are mean \pm SD or median (interquartile range) unless otherwise indicated

${ }^{a}$ With a parent or first-degree sibling with any of the following conditions: coronary heart disease, stroke, hypertension, or diabetes

${ }^{\mathrm{b}} n=327$ because of missing value

replacing CRP with PAI-1 (RR 1.30; 95\% CI 1.06, 1.59) or palmitoleic acid (RR 1.26; 95\% CI 1.03, 1.55) in Model 3 , there was little impact on the association. Among five components of MetS, incidences of hypertriacylglycerolaemia and hyperglycaemia were significantly associated with baseline plasma LBP across all the models (Table 3).

In stratified analysis by BMI categories, LBP was associated with an increased risk of MetS only in normal-weight individuals (RR, comparing extreme tertiles, $1.59 ; 95 \% \mathrm{CI}$ $1.18,2.15 ; p$ trend $=0.002$ ), but not in those who were overweight/obese (RR, comparing extreme tertiles, 0.99;
$95 \%$ CI $0.80,1.22 ; p$ trend $=0.880)$. A significant interaction was observed between LBP and obesity status ( $p$ interaction $=$ 0.013 ). When the data were stratified according to the median of BMI, the positive association between LBP and incident MetS was only evidenced in individuals with a $\mathrm{BMI}<22.7$ $\mathrm{kg} / \mathrm{m}^{2}$, but not in those with a BMI $\geq 22.7 \mathrm{~kg} / \mathrm{m}^{2}$ ( $p_{\text {interaction }}=$ 0.051) (Table 4). Moreover, when sex was taken into account, the positive association remained significant in both men and women (Table 4). No significant interactions were detected when stratified analyses were performed according to age, sex and CRP levels (all $p_{\text {interaction }}>0.05$ ). 
Table 2 Partial Spearman correlation coefficients between baseline LBP and metabolic traits at baseline and follow-up

\begin{tabular}{|c|c|c|c|c|c|c|}
\hline \multirow[t]{2}{*}{ Variable } & \multicolumn{2}{|l|}{$\begin{array}{l}\text { Total } \\
(n=1,312)\end{array}$} & \multicolumn{2}{|c|}{$\begin{array}{l}\mathrm{BMI}<24 \mathrm{~kg} / \mathrm{m}^{2} \\
(n=875)\end{array}$} & \multicolumn{2}{|c|}{$\begin{array}{l}\mathrm{BMI} \geq 24 \mathrm{~kg} / \mathrm{m}^{2} \\
(n=437)\end{array}$} \\
\hline & Baseline & Follow-up & Baseline & Follow-up & Baseline & Follow-up \\
\hline BMI & $0.109 * * *$ & $0.075^{*}$ & $0.075^{*}$ & 0.042 & 0.029 & -0.008 \\
\hline WC & $0.120 * * *$ & $0.097 * * *$ & $0.075^{*}$ & $0.072 *$ & 0.069 & 0.028 \\
\hline Glucose & -0.027 & $0.076^{*}$ & -0.016 & $0.077^{*}$ & -0.063 & 0.053 \\
\hline $\mathrm{HbA}_{1 \mathrm{c}}$ & $0.096^{*}$ & 0.053 & $0.102 *$ & 0.057 & 0.069 & 0.028 \\
\hline Triacylglycerol & $0.091^{*}$ & $0.087^{*}$ & $0.073^{*}$ & $0.108^{*}$ & 0.093 & -0.006 \\
\hline Total cholesterol & 0.032 & $0.056^{*}$ & -0.021 & 0.034 & $0.121^{*}$ & 0.080 \\
\hline HDL-cholesterol & $-0.090 *$ & $-0.058^{*}$ & $-0.119 * * *$ & $-0.088 *$ & -0.005 & 0.037 \\
\hline LDL-cholesterol & 0.049 & $0.066^{*}$ & 0.010 & 0.042 & $0.109^{*}$ & 0.089 \\
\hline Triacylglycerol/HDL-cholesterol ratio & $0.111 * * *$ & $0.088^{*}$ & $0.107 *$ & $0.123 * * *$ & 0.080 & -0.037 \\
\hline Systolic blood pressure & 0.039 & $0.065^{*}$ & 0.04 & $0.081^{*}$ & 0.010 & 0.015 \\
\hline Diastolic blood pressure & 0.006 & $0.060^{*}$ & -0.004 & 0.047 & -0.002 & 0.062 \\
\hline CRP & $0.389^{* * *}$ & $0.218^{* * *}$ & $0.398 * * *$ & $0.216^{* * *}$ & $0.351 * * *$ & $0.197 * * *$ \\
\hline Insulin $^{\mathrm{a}}$ & 0.023 & 0.053 & 0.031 & 0.057 & -0.041 & -0.023 \\
\hline HOMA-IR ${ }^{\mathrm{a}}$ & 0.019 & $0.058^{*}$ & 0.027 & 0.060 & -0.044 & -0.017 \\
\hline
\end{tabular}

Values are adjusted for age, sex, region and residence

${ }^{\text {a }} n=1,306, n=871$ and $n=435$, respectively, because of missing values

${ }^{*} p<0.05 ; * * p<0.001$

\section{Discussion}

To the best of our knowledge, this is the first population-based prospective study of the relationship between plasma LBP levels and metabolic conditions. Our study found that LBP was significantly associated with increased 6 year risks of MetS, hypertriacylglycerolaemia and hyperglycaemia, independently of established risk factors including BMI and CRP, in a middle-aged and older Chinese population. Moreover, we found that the LBP-MetS association was more pronounced

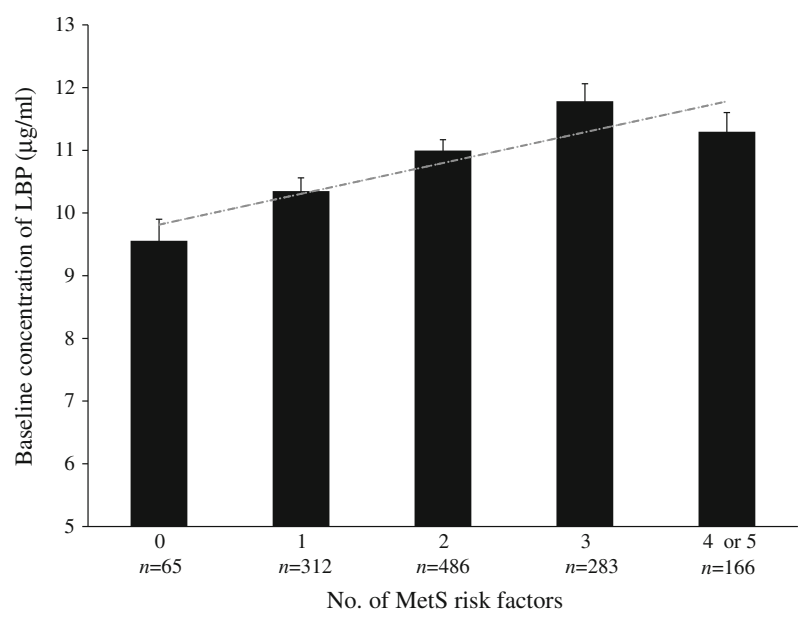

Fig. 1 Baseline concentration of LBP according to number of MetS risk factors at follow-up in $2011(p<0.001)$ in normal-weight participants than in overweight and obese individuals.

Since its discovery in the 1980s, LBP has been considered a key protein in mediating LPS-triggered innate immunity in a CD14- and TLR4-dependent manner [7, 20]. As a surrogate marker of LPS, circulating LBP concentration was shown to be positively associated with prevalence of MetS in our previous overweight/obese case-control analysis [9]. Likewise, a recent cross-sectional study in 420 Spanish individuals also indicated that higher LBP was associated with MetS and its components, including central obesity and low HDL-cholesterol [12]. However, the cross-sectional nature of previous studies could lead to reverse causation, since common symptoms of MetS may also alter gastrointestinal conditions and promote occurrence of subclinical endotoxaemia $[10,11]$. With a prospective design, our current analyses provided stronger evidence to support the putative contributions of endotoxaemia to the development of metabolic abnormalities.

Both hyperglycaemia and hypertriacylglycerolaemia have been observed in animals following LPS infusion [21-23], as well as in patients with sepsis or other infectious disorders [24, 25]. Our study, at a population level, indicated that exposure to increased LBP may contribute to increased 6 year incidence of hyperglycaemia and hypertriacylglycerolaemia, the latter showing the strongest association with baseline LBP levels among all of the MetS components. In our previous cross-sectional analysis, the OR of prevalent hypertriacylglycerolaemia comparing extreme quartiles of LBP was also much higher than the 
Table 3 RR (95\% CI) of MetS and its components in 6 year follow-up according to quartiles (Q) of baseline LBP

\begin{tabular}{|c|c|c|c|c|c|}
\hline MetS/component of MetS & Q1 & Q2 & Q3 & Q4 & $p$ value trend \\
\hline Metabolic syndrome & $93 / 328$ & $108 / 328$ & $109 / 328$ & $139 / 328$ & \\
\hline Model 1 & 1 & $1.18(0.93,1.48)$ & $1.24(0.98,1.56)$ & $1.52(1.23,1.89)$ & $<0.001$ \\
\hline Model 2 & 1 & $1.15(0.93,1.42)$ & $1.08(0.87,1.34)$ & $1.30(1.06,1.59)$ & 0.023 \\
\hline Model 3 & 1 & $1.14(0.92,1.42)$ & $1.07(0.86,1.34)$ & $1.28(1.04,1.58)$ & 0.046 \\
\hline Central obesity & $54 / 287$ & $64 / 288$ & $62 / 288$ & $68 / 288$ & \\
\hline Model 1 & 1 & $1.23(0.89,1.70)$ & $1.28(0.93,1.78)$ & $1.39(1.01,1.91)$ & 0.044 \\
\hline Model 2 & 1 & $1.24(0.92,1.68)$ & $1.24(0.91,1.68)$ & $1.24(0.93,1.67)$ & 0.178 \\
\hline Model 3 & 1 & $1.25(0.92,1.68)$ & $1.24(0.91,1.68)$ & $1.25(0.92,1.70)$ & 0.180 \\
\hline Hypertriacylglycerolaemia & $91 / 425$ & $95 / 426$ & $120 / 426$ & $147 / 425$ & \\
\hline Model 1 & 1 & $1.04(0.81,1.35)$ & $1.34(1.06,1.71)$ & $1.59(1.27,2.00)$ & $<0.001$ \\
\hline Model 2 & 1 & $1.02(0.79,1.31)$ & $1.25(0.99,1.59)$ & $1.45(1.15,1.82)$ & $<0.001$ \\
\hline Model 3 & 1 & $1.02(0.79,1.31)$ & $1.26(0.99,1.60)$ & $1.46(1.16,1.86)$ & $<0.001$ \\
\hline Hyperglycaemia & $252 / 335$ & $259 / 337$ & $262 / 337$ & $282 / 336$ & \\
\hline Model 1 & 1 & $1.04(0.95,1.13)$ & $1.06(0.97,1.15)$ & $1.12(1.04,1.21)$ & 0.003 \\
\hline Model 2 & 1 & $1.03(0.95,1.12)$ & $1.04(0.96,1.13)$ & $1.10(1.02,1.19)$ & 0.015 \\
\hline Model 3 & 1 & $1.03(0.94,1.12)$ & $1.04(0.96,1.13)$ & $1.09(1.01,1.18)$ & 0.031 \\
\hline Elevated blood pressure & $56 / 175$ & $61 / 175$ & $62 / 175$ & $63 / 175$ & \\
\hline Model 1 & 1 & $1.00(0.74,1.35)$ & $1.00(0.74,1.33)$ & $1.07(0.81,1.43)$ & 0.644 \\
\hline Model 2 & 1 & $1.01(0.75,1.35)$ & $0.97(0.72,1.30)$ & $1.02(0.77,1.36)$ & 0.943 \\
\hline Model 3 & 1 & $0.98(0.73,1.32)$ & $0.94(0.70,1.27)$ & $0.95(0.70,1.29)$ & 0.692 \\
\hline Low HDL-cholesterol & $36 / 328$ & $44 / 326$ & $27 / 327$ & $56 / 326$ & \\
\hline Model 1 & 1 & $1.19(0.79,1.80)$ & $0.76(0.47,1.23)$ & $1.57(1.06,2.31)$ & 0.096 \\
\hline Model 2 & 1 & $1.16(0.76,1.75)$ & $0.70(0.43,1.14)$ & $1.43(0.96,2.13)$ & 0.234 \\
\hline Model 3 & 1 & $1.12(0.74,1.68)$ & $0.66(0.41,1.08)$ & $1.27(0.83,1.93)$ & 0.607 \\
\hline
\end{tabular}

Model 1, adjusted for age, sex, region (north, south), residence (urban, rural), current smoking (yes, no), current drinking (yes, no), educational level (06 years, 7-9 years, $\geq 10$ years), physical activity (low, moderate, high) and family history of chronic diseases (yes, no); Model 2, further adjusted for BMI; Model 3, further adjusted for baseline CRP

rest of the MetS components [9]. However, the mechanism(s) underlying the LBP-hypertriacylglycerolaemia association remain(s) largely unknown. Sharing sequence identity between LBP and lipid transfer proteins may modify lipid homeostasis during subclinical endotoxaemia [26]. Low-dose treatment of LPS in rodent models was found to stimulate hepatic de novo lipogenesis (DNL) and lipolysis and, consequently, increased liver production of triacylglycerol [22]. In humans, erythrocyte palmitoleic acid has been suggested as a biomarker of DNL [27] and was significantly correlated with plasma LBP in the current study (ESM Table 2). Indeed, palmitoleic acid per se was found to be independently associated with a 6 year risk of hypertriacylglycerolaemia in our earlier analysis [14]. However, controlling for erythrocyte palmitoleic acid attenuated the LBP-hypertriacylglycerolaemia correlation (at baseline, from $r=0.091$ to $r=0.067$; at follow-up visit, from $r=0.087$ to $r=0.070$ ), suggesting that the effect of LBP on dyslipidaemia might be somewhat explained by enhanced DNL.

As a well-established metabolic risk factor, obesity has been considered a chronic inflammatory condition [28], while the LPS/LBP-triggered extrinsic inflammatory cascade seems also to play a role in the pathogenesis of metabolic disorders [20]. Thus, we examined the relationships of LBP with BMI and CRP on the MetS risk in stratified analyses. It was interesting that the LBP-MetS association was only significant in normal-weight participants, but not in their overweight/obese counterparts $(p$ interaction $=0.013)$, despite higher prevalence of having two MetS components at baseline (ESM Fig. 1). It is plausible that the effect of LBP on the association might be confounded by co-existing metabolic abnormalities in overweight/obese individuals. On the other hand, Shah et al recently observed that LPS-treated non-obese healthy people had suppressed expression of adipose genes involving cell development and differentiation [29]. They also found that most of those genes ( 20 out of 22 ) in adipose tissue were downregulated in obese individuals. In other words, LPS invasion in those who are not obese might have an obesogenic-like impact on regulating gene expression. Moreover, Mehta and co-workers reported that experimental endotoxaemia by LPS infusion in non-obese men and women could induce systemic inflammation and insulin resistance [5]. Notably, circulating LBP in the current study was closely 
Table 4 Stratified analysis of RR (95\% CI) of MetS in 6 year follow-up according to tertiles (T) of baseline LBP

\begin{tabular}{|c|c|c|c|c|c|}
\hline Variable & $\mathrm{T} 1$ & $\mathrm{~T} 2$ & $\mathrm{~T} 3$ & $p$ value ${ }_{\text {trend }}$ & $p$ value interaction \\
\hline Age & & & & & 0.697 \\
\hline $50-59$ years & 1 & $1.38(1.08,1.76)$ & $1.38(1.08,1.77)$ & 0.008 & \\
\hline 60-70 years & 1 & $1.03(0.75,1.40)$ & $1.26(0.94,1.69)$ & 0.104 & \\
\hline Age (median) & & & & & 0.253 \\
\hline $50-56$ years & 1 & $1.26(0.96,1.65)$ & $1.19(0.91,1.56)$ & 0.191 & \\
\hline $57-70$ years & 1 & $1.19(0.91,1.57)$ & $1.46(1.12,1.90)$ & 0.004 & \\
\hline Sex & & & & & 0.686 \\
\hline Men & 1 & $1.03(0.74,1.44)$ & $1.38(1.03,1.85)$ & 0.027 & \\
\hline Women & 1 & $1.34(1.05,1.70)$ & $1.29(1.01,1.64)$ & 0.042 & \\
\hline BMI & & & & & 0.013 \\
\hline$<24 \mathrm{~kg} / \mathrm{m}^{2}$ & 1 & $1.32(0.97,1.81)$ & $1.59(1.18,2.15)$ & 0.002 & \\
\hline$\geq 24 \mathrm{~kg} / \mathrm{m}^{2}$ & 1 & $1.04(0.84,1.28)$ & $0.99(0.80,1.22)$ & 0.880 & \\
\hline BMI (median) & & & & & 0.051 \\
\hline$<22.7 \mathrm{~kg} / \mathrm{m}^{2}$ & 1 & $1.35(0.91,2.00)$ & $1.62(1.10,2.39)$ & 0.013 & \\
\hline$\geq 22.7 \mathrm{~kg} / \mathrm{m}^{2}$ & 1 & $1.04(0.85,1.28)$ & $1.05(0.87,1.28)$ & 0.600 & \\
\hline CRP (median) & & & & & 0.877 \\
\hline$<0.45 \mathrm{mg} / 1$ & 1 & $1.26(0.94,1.69)$ & $1.16(0.80,1.67)$ & 0.294 & \\
\hline$\geq 0.45 \mathrm{mg} / 1$ & 1 & $1.08(0.84,1.39)$ & $1.15(0.91,1.45)$ & 0.235 & \\
\hline
\end{tabular}

Values are adjusted for age, sex, region (north, south), residence (urban, rural), current smoking (yes, no), current drinking (yes, no), educational level (06 years, 7-9 years, $\geq 10$ years), physical activity (low, moderate, high), family history of chronic diseases (yes, no)

correlated with CRP levels independently of adiposity level. Controlling for BMI and CRP did not materially change the significant association between LBP and incident MetS, suggesting that moderate endotoxaemia or elevated LBP might initiate an innate immune response and introduce inflammatory stress and metabolic dysfunction through mechanisms which do not fully overlap with the obesity-mediated pathway. LPS injection in lean mice induced a unique inflammatory response, characterised by elevated hepatic expression of PAI-1 and circulating galectin-3 [30], which may modify metabolic homeostasis; supporting evidence from humans was, however, limited. Taken together, the findings from the current study highlight the critical role of subclinical endotoxaemia, indicated by elevated circulating LBP, on future metabolic deterioration in normal-weight participants over several years.

The present study has valuable strengths: (1) a longitudinal study design enabled us to discuss temporal relationships between exposure to LBP and incident MetS; (2) a number of potential confounders or mediators were considered and carefully controlled. Admittedly, our study had several limitations. First, participants were aged over 50 years at baseline, and it remains unknown whether our results could be generalised to other age groups. Second, MetS was newly defined at the follow-up visit; thus, it was not possible to include the exact disease-free duration in our analysis. Finally, due to the limited number of overweight/obese individuals after stratification, the observed interaction between BMI and LBP might have been due to chance and should be replicated in further studies.
In conclusion, our study indicated that elevated plasma LBP was positively associated with 6 year MetS incidence, especially among normal-weight individuals. Future studies are needed to corroborate our findings and to elucidate the potential biological mechanisms.

Acknowledgements We express our sincere appreciation to J. Zhu, G. Liu, Y. Hu, H. Zheng and Y. Wang, at the Institute for Nutritional Sciences, Shanghai Institutes for Biological Sciences, Chinese Academy of Sciences and University of Chinese Academy of Sciences, for their assistance in this study.

Funding The study was supported by the Ministry of Science and Technology of China (2012CB524900), National Natural Science Foundation of China $(81202272,81321062,30930081,81200581$ and 81170734), the Knowledge Innovation Program of Shanghai Institutes for Biological Sciences, Chinese Academy of Sciences (2013KIP107), and the SA-SIBS Scholarship Program. These funding sources had no role in any aspect of the study, including the study design, data collection, data analysis and interpretation of results.

Duality of interest The authors declare that there is no duality of interest associated with this manuscript.

Contribution statement X. Liu and LL contributed to the study design, data collection and data analyses, and wrote the manuscript. PY, YM, FW, QJ, XY and HL contributed to the study design, data collection and editing of the manuscript. FBH contributed to the interpretation of the data and editing of the manuscript. LS contributed to the study design, data collection, interpretation of the data and editing of the manuscript. X. Lin contributed to the study design, data collection, discussion and editing of the manuscript. All authors approved the manuscript before submission. X. Lin is the guarantor of this work. 


\section{References}

1. Zhao L (2013) The gut microbiota and obesity: from correlation to causality. Nat Rev Microbiol 11:639-647

2. Vijay-Kumar M, Aitken JD, Carvalho FA et al (2010) Metabolic syndrome and altered gut microbiota in mice lacking Toll-like receptor 5. Science 328:228-231

3. Qin J, Li Y, Cai Z et al (2012) A metagenome-wide association study of gut microbiota in type 2 diabetes. Nature 490:55-60

4. Kemp DM (2013) Does chronic low-grade endotoxemia define susceptibility of obese humans to insulin resistance via dietary effects on gut microbiota? Adipocyte 2:188-190

5. Mehta NN, McGillicuddy FC, Anderson PD et al (2010) Experimental endotoxemia induces adipose inflammation and insulin resistance in humans. Diabetes 59:172-181

6. Novitsky TJ (1998) Limitations of the Limulus amebocyte lysate test in demonstrating circulating lipopolysaccharides. Ann N Y Acad Sci 851:416-421

7. Schumann RR (2011) Old and new findings on lipopolysaccharidebinding protein: a soluble pattern-recognition molecule. Biochem Soc Trans 39:989-993

8. Ruiz AG, Casafont F, Crespo J et al (2007) Lipopolysaccharide-binding protein plasma levels and liver TNF-alpha gene expression in obese patients: evidence for the potential role of endotoxin in the pathogenesis of non-alcoholic steatohepatitis. Obes Surg 17:1374-1380

9. Sun L, Yu Z, Ye X et al (2010) A marker of endotoxemia is associated with obesity and related metabolic disorders in apparently healthy Chinese. Diabetes Care 33:1925-1932

10. Teixeira TF, Souza NC, Chiarello PG et al (2012) Intestinal permeability parameters in obese patients are correlated with metabolic syndrome risk factors. Clin Nutr 31:735-740

11. Manco M, Putignani L, Bottazzo GF (2010) Gut microbiota, lipopolysaccharides, and innate immunity in the pathogenesis of obesity and cardiovascular risk. Endocr Rev 31:817-844

12. Gonzalez-Quintela A, Alonso M, Campos J, Vizcaino L, Loidi L, Gude F (2013) Determinants of serum concentrations of lipopolysaccharide-binding protein (LBP) in the adult population: the role of obesity. PLoS One 8:e54600

13. Moreno-Navarrete JM, Escote X, Ortega F et al (2013) A role for adipocyte-derived lipopolysaccharide-binding protein in inflammation- and obesity-associated adipose tissue dysfunction. Diabetologia 56:2524-2537

14. Zong G, Zhu J, Sun L et al (2013) Associations of erythrocyte fatty acids in the de novo lipogenesis pathway with risk of metabolic syndrome in a cohort study of middle-aged and older Chinese. Am J Clin Nutr 98:319-326

15. Sun L, Zong G, Pan A et al (2013) Elevated plasma ferritin is associated with increased incidence of type 2 diabetes in middleaged and elderly Chinese adults. J Nutr 143:1459-1465
16. Yu Z, Ye X, Wang J et al (2009) Associations of physical activity with inflammatory factors, adipocytokines, and metabolic syndrome in middle-aged and older chinese people. Circulation 119:2969-2977

17. Zhou BF (2002) Predictive values of body mass index and waist circumference for risk factors of certain related diseases in Chinese adults - study on optimal cut-off points of body mass index and waist circumference in Chinese adults. Biomed Environ Sci 15:83-96

18. Grundy SM, Cleeman JI, Daniels SR et al (2005) Diagnosis and management of the metabolic syndrome: an American Heart Association/National Heart, Lung, and Blood Institute scientific statement. Circulation 112:2735-2752

19. Zou G (2004) A modified Poisson regression approach to prospective studies with binary data. Am J Epidemiol 159:702-706

20. Cani PD, Amar J, Iglesias MA et al (2007) Metabolic endotoxemia initiates obesity and insulin resistance. Diabetes 56:1761-1772

21. Bartolome N, Aspichueta P, Martinez MJ et al (2012) Biphasic adaptative responses in VLDL metabolism and lipoprotein homeostasis during Gram-negative endotoxemia. Innate Immun 18:89-99

22. Feingold KR, Staprans I, Memon RA et al (1992) Endotoxin rapidly induces changes in lipid metabolism that produce hypertriglyceridemia: low doses stimulate hepatic triglyceride production while high doses inhibit clearance. J Lipid Res 33:1765-1776

23. Sugita H, Kaneki M, Tokunaga E et al (2002) Inducible nitric oxide synthase plays a role in LPS-induced hyperglycemia and insulin resistance. Am J Physiol Endocrinol Metab 282:E386-E394

24. Gornik I, Vujaklija A, Lukic E, Madzarac G, Gasparovic V (2010) Hyperglycemia in sepsis is a risk factor for development of type II diabetes. J Crit Care 25:263-269

25. Visschers RG, Olde Damink SW, Schreurs M, Winkens B, Soeters PB, van Gemert WG (2009) Development of hypertriglyceridemia in patients with enterocutaneous fistulas. Clin Nutr 28:313-317

26. Hudgins LC, Parker TS, Levine DM et al (2003) A single intravenous dose of endotoxin rapidly alters serum lipoproteins and lipid transfer proteins in normal volunteers. J Lipid Res 44:1489-1498

27. Zong G, Ye X, Sun L et al (2012) Associations of erythrocyte palmitoleic acid with adipokines, inflammatory markers, and the metabolic syndrome in middle-aged and older Chinese. Am J Clin Nutr 96:970-976

28. Fantuzzi G (2005) Adipose tissue, adipokines, and inflammation. J Allergy Clin Immunol 115:911-919, quiz 920

29. Shah R, Hinkle CC, Haris L, Mehta NN, Putt ME, Reilly MP (2012) Adipose genes down-regulated during experimental endotoxemia are also suppressed in obesity. J Clin Endocrinol Metab 97:E2152-E2159

30. Pini M, Castellanos KJ, Rhodes DH, Fantuzzi G (2013) Obesity and IL-6 interact in modulating the response to endotoxemia in mice. Cytokine 61:71-77 